\title{
Gingival Changes in Wistar Rats after Oral Treatment with 4-Nitroquinoline 1-Oxide
}

Daniel Araki Ribeiroa,

Daisy Maria Fávero Salvadori ${ }^{b}$

\begin{abstract}
Objectives: 4-nitroquinoline 1-oxide (4NQO)-induced rat tongue carcinogenesis is a useful model for studying oral squamous cell carcinoma. However, gingival changes following 4NQO administration via drinking water are absent in the literature. The aim of this study was to investigate gingival changes concomitant to tongue carcinogenesis induced by $4 \mathrm{NQO}$ by means of morphological analysis.

Methods: Male Wistar rats were distributed into 3 groups of 10 animals each and treated with 50 ppm 4NQO solution by drinking water for 4, 12 or 20 weeks. Thirty animals were used as negative control.

Results: Regarding tongue mucosa, the primary histopathological change i.e., hyperplasia and dysplasia was evidenced after 12 weeks treatment with 4 NQO. At 20 weeks, squamous cell carcinoma was found in the majority of animals. Gingival squamous hyperplasia was induced by $4 \mathrm{NQO}$ after 20-weeks of treatment. Dysplastic changes appeared in some animals (two cases) as well.

Conclusions: Taken together, our results support the notion that $4 \mathrm{NQO}$ is more effective in rat tongue mucosa than gingival tissue. Probably, this discrepancy depends strongly on route of administration and the susceptibility with respect to animals species. Certainly, such data will contribute when using this experimental test-system for understanding oral cancer pathogenesis. (Eur J Dent 2007; 1:152-157)
\end{abstract}

Keywords: Oral cancer; 4-nitroquinoline 1-oxide; Rat; Gingiva.

\section{INTRODUCTION}

Oral cancer is a common neoplasm worldwide, particularly in developing countries such as India, Vietnam and Brazil, where it constitutes up to $25 \%$ of all types of cancer. ${ }^{1}$ Despite of the sophisticated surgical and radiotherapeutic modalities, the patient survival has not improved significantly during the last decades. ${ }^{2}$ Tobacco and alcohol consump-

Department of Health Sciences,

Federal University of Sao Paulo, UNIFESP, SP, Brazil;

b Department of Pathology, Botucatu Medical School,

Sao Paulo State University, UNESP, SP, Brazil

- Corresponding Author: Daniel Araki Ribeiro, DDS, PhD Departamento de Ciências da Saúde Av. Ana Costa, 95 Vila Mathias Santos - SP, 11060-001, Brazil Phone : 551332222048

Fax : 551332222048 e-mail: daribeirodunifesp.br tion are the most significant exogenous factors involved in tumorigenesis. ${ }^{3}$

The most used animal models in oral cancer research are the hamster buccal pouch by fatsoluble 7,12 dimethylbenzanthracene (DMBA), and the rat tongue by water-soluble 4-nitroquinoline 1-oxide (4NQO). ${ }^{4}$ Considering that one of the most important routes of oral carcinogens is through liquid containing water-soluble carcinogens, $4 \mathrm{NQO}$ is well suited in examining the role of xenobiotics in experimental oral carcinogenesis. ${ }^{5}$ Based on the multi-step process of carcinogenesis characterized by initiation, promotion and tumor progression, chronic administration of $4 \mathrm{NQO}$ in drinking water simulates rat tongue carcinogenesis like human counterpart.6-10 
Moreover, oral administration of $4 \mathrm{NQO}$ produces tumor in other tissues of oral cavity as well as aerodigestive tract. ${ }^{11}$ For example, some researchers have described changes induced by $4 N Q 0$ intake in minor salivary glands ${ }^{10}$ and esophageal mucosa. ${ }^{11}$ However, to our knowledge, there are no studies reporting gingival changes during tongue carcinogenesis induced by $4 \mathrm{NQO}$ incorporated into drinking water so far. As a result of limited evidence, this study was aimed to look at putative changes on gingival tissue concomitant to tongue carcinogenesis following systemic exposure to $4 \mathrm{NQO}$. Certainly, such data will contribute to a better understanding of this mediumterm oral carcinogenesis assay currently used for studying oral cancer pathogenesis.

\section{MATERIAL AND METHODS}

Animals and experimental design

A total of 60 male Wistar rats ( 8 weeks old) weighting approximately $250 \mathrm{~g}$, were obtained from Centro de Bioterismo (CEMIB), Universidade Estadual de Campinas, SP, Brazil. They were maintained under controlled conditions of temperature $\left(24 \pm 2^{\circ} \mathrm{C}\right.$ ), light-dark periods of 12 hours, and with free access to water and commercial diet (Nuvital PR, Brazil). They were treated with 50 ppm 4NQO (Sigma, St. Louis, MO, USA) solution by drinking water for 4, 12 and 20 weeks. Thirty animals were used as negative control. At the end of the experimental period, the rats were sacrificed by $0.4 \%$ sodium pentobarbital $(1 \mathrm{~mL} / \mathrm{kg}$ body wt, i.p.). The final body weight and weight gain were recorded. The tongues and mandibles were longitudinally cut into halves for histopathological examinations. For this, the mandibles were decalcified in a $5 \%$ of nitric acid solution (Merck, Darmstadt, Germany) for 5 days. After fixation and/or decalcification, all of the tissues were trimmed, dehydrated, cleared, embedded in paraffin, sectioned into $5 \mu \mathrm{m}$ tick section, stained with hematoxylin and eosin (H.E.) All experimental protocols were approved by the Animal Committee the Botucatu Medical School, UNESP, Brazil.

\section{Histopathological analysis}

Histopathological evaluation was performed under light microscope. Analyzes of the tongue and mandible sections in the molar tooth areas were graded as normal, hyperplasia, dysplasia and carcinoma by Kramer et al. ${ }^{12}$

\section{Statistical analysis}

Results from the final body weight and weight gain were analyzed by one way ANOVA, followed by Tukey's test. The level of significance was set at $5 \%$.

\section{RESULTS}

Quantitative results

Final body weight and weight gain during the experimental period are presented in Table 1. Excluding 12 weeks treated group for final body weight, all groups showed statistically decreases $(P<.05)$ either to final body weight or to weight gain when compared to control group.

\section{Morphological results}

The dorsal surface of the rats' tongue treated with $4 \mathrm{NQO}$ became white with a rough appearance after 12 weeks-treatment only. As time progressed, ulcers were found in the same region in the majority of animals. No apparent abnormal clinical signs were noticed to gingival tissue in all periods evaluated.

Regarding subjective microscopic analysis, no histopathological changes in tongue epithelia were observed in the control group (Figure 1a) nor

Table 1. Mean \pm Standard deviation of final body weight and weight gain of rats in the 4-nitroquinoline 1-oxide (4NQO)a model for oral carcinogenesis.

\begin{tabular}{|c|c|c|c|c|c|c|}
\hline \multirow[t]{2}{*}{ Dose (ppm) } & \multicolumn{2}{|c|}{4 weeks } & \multicolumn{2}{|c|}{12 weeks } & \multicolumn{2}{|c|}{20 weeks } \\
\hline & $\begin{array}{c}\text { Final body } \\
\text { weight }\end{array}$ & Weight gain & $\begin{array}{c}\text { Final body } \\
\text { weight }\end{array}$ & Weight gain & $\begin{array}{c}\text { Final body } \\
\text { weight }\end{array}$ & Weight gain \\
\hline 0 & $341.56 \pm 27.4$ & $45.16 \pm 3.12$ & $396.31 \pm 54.59$ & $99.91 \pm 10.57$ & $462.3 \pm 23.46$ & $165.9 \pm 18.57$ \\
\hline 50 & $273.52 \pm 26.5^{*}$ & $6.66 \pm 1.04^{*}$ & $314.78 \pm 34.34$ & $34.6 \pm 5.87^{*}$ & $377.2 \pm 60.13^{*}$ & $97.2 \pm 20.48^{*}$ \\
\hline
\end{tabular}

$*: P<.05$ when compared to control group (zero).

a 4 NQO - 50 ppm by drinking water 
after $4^{\text {th }}$ week treatment with $4 \mathrm{NQO}$. The primary histopathological change, i.e., hyperplasia and hyperkeratosis with the spinous cell layer gradually tickned was evidenced after 12-weeks-treatment (Figure 1b). In this period, epithelial dysplasia was also found in mild and moderate forms (Figure 1c). At 20 weeks, moderate and/or severe oral dysplasia (Figure 1c) and squamous cell carcinoma in the tongue (Figure 1d) were found; being that in the majority of animals consisted of squamous cell carcinoma. The histopathological grade of the carcinomas was usually squamous cell carcinoma of a well-differentiated type. The tumors spread into the submucosa and underlying muscle layer, forming small nests with typical keratin pearl formation. In advanced cases, severe atypia was frequently found. The histopathological findings are summarized in the Table 2.

Finally, no significant changes were observed until 12-weeks of treatment with $4 \mathrm{NQO}$ in the gingival mucosa (Figure 2a). However, cases showing the presence of coarse food particles upon gingival mucosa producing inflammation in the periodontal tissue adjacent the molar tooth were noted sporadically in control and experimental animals (Figure 2b). No significant differences in the incidences of gingivitis occurred between control and experimental groups (data not shown). Gingival squamous hyperplasia induced by $4 \mathrm{NQO}$

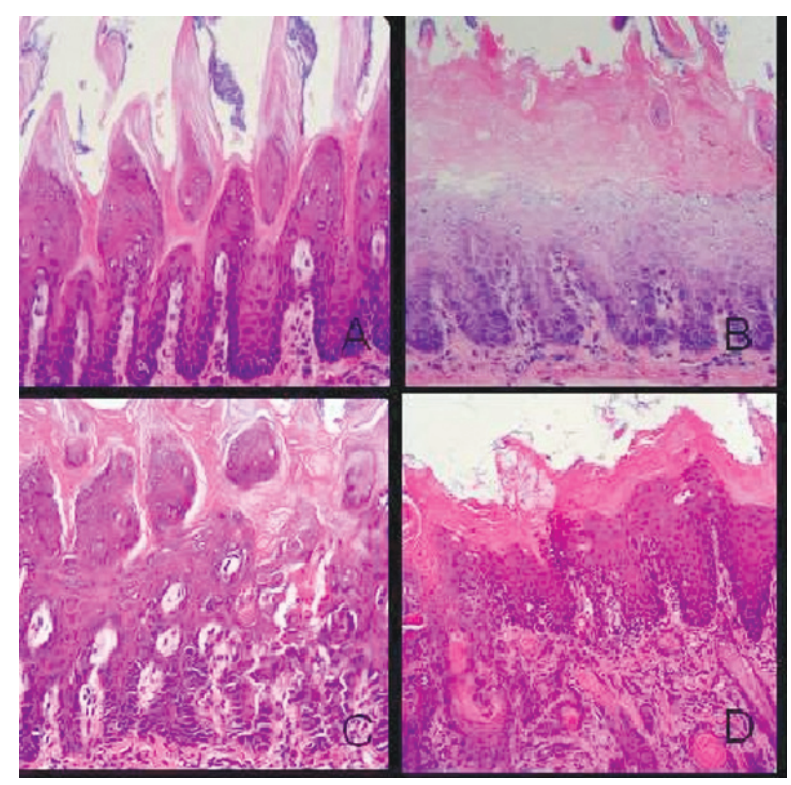

Figure 1. Photomicrographies showing the multi-step process of rat tongue carcinogenesis. (A) no histopathological change (control); (B) hyperplasia and hyperkeratosis; (C) epithelial dysplasia; (D) squamous cell carcinoma of well-differentiated type. (Hematoxylin and Eosin stain; X 100 magnification). after 20-weeks of treatment constituted a focal lesion that manifested itself in the stratified squamous epithelium of oral mucosa adjacent to molar tooth. It consisted of varying degrees of thickening of the epithelium showing hyperkeratosis, often with the formation of epithelial rete pegs that extended into the underlying connective tissue (Figure 2c). Endophytic proliferation of tissue gingival squamous hyperplasia occurred most commonly rather than exophytic projections in the stratified squamous epithelium. The proliferative cells producing keratin displayed prominent intercellular bridges and resembled normal stratified squamous epithelium. In this group, dysplastic changes appeared in some animals (two cases) as well. The dysplastic cells contained large nuclei with clear nucleoli, eosinophilic cytoplasm, mitotic figures, and coexistent dyskeratotic, suggesting cellular atypia (Figure $2 \mathrm{~d}$ ). All findings are summarized in Table 3.

\section{DISCUSSION}

The aim of chemical carcinogenesis models is to provide further understanding concerning to the multi-step process of carcinogenesis in order to investigate hazard risk caused by environmental agents as well as to develop strategies to earlier diagnostic and/or cancer prevention. Several medium-term duration assay systems for oral carci-
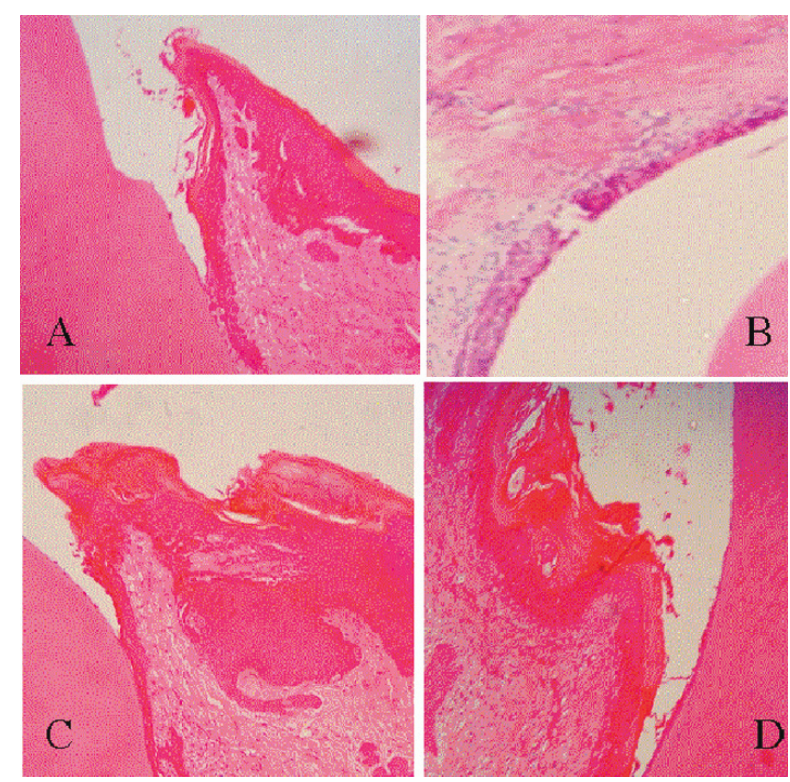

Figure 2. Photomicrographies of gingival tissue from control group and that treated with $4 \mathrm{NQO}$. (A) control group, (B) control group presenting inflammation (C) hyperplasia and (C) dysplasia. (Hematoxylin and Eosin stain; X 100 magnification). 
nogenesis offer particular promise. One of these models is DMBA-induced carcinoma produced in the hamster cheek pouch. Because of its peculiar anatomic site and histology, some authors have questioned the suitability of the hamster cheek pouch for studies related to oral tumors. ${ }^{13}$ In this regard, the Wistar rat is a good alternative because it is ease of handling and availability. Furthermore, $4 N Q 0$-induced rat tongue tumors are more realistic since reveal histological and biochemical characteristics similar to the human tumors. ${ }^{6-10}$ Such results obtained from this present investigation, using $4 N Q O$ as carcinogen inducers, demonstrated histopathological changes in tongue mucosa along a time-course experiment from normal epithelium, hyperplasia and hyperkeratosis, pre-malignant dysplasia, and carcinoma in situ to invasive squamous cell carcinoma. ${ }^{14,15}$

Accumulating evidence suggests that diagnosis of human gingival squamous cell carcinoma is prone to delay because it is rare, and thus unexpected, and because it may be mistaken clinically for gingival or periodontal infection. ${ }^{16}$ Gingival carcinoma usually occurs in the lower jaw in posterior sites with pain, swelling and loosening of adjacent teeth being common clinical signs. ${ }^{16}$ For this reason, gingival lesions were investigated herein within the areas of the lower molar-tooth ligament (gingival sulcus). In the rat, the gingiva consists of a keratinized stratified squamous epithelium, connective tissue with fibroblasts, and the extracellular matrix, composed chiefly of collagen fibers and ground substance containing sulfated glycosaminoglucans. ${ }^{17-19}$ The gingival epithelium in the molar-tooth area is classified as gingival oral epithelium, sulcular epithelium and junctional epithelium, which appears non-keratinized and forms the floor of the gingival sulcus. Gingival epithelium manifests a higher proliferative capacity and higher rate of absorption of drugs and chemicals than the skin. ${ }^{18,20}$ Mitotic activity appears greatest at the dento-gingival junction of incisors, especially within the junctional epithelium. ${ }^{21,22}$ It is important to stress that configuration of gingival crevice of molar in rat is different from it of human. Therefore, any extrapolation with respect to gingival carcinogenesis in rodents should be interpreted catiously.

Taking into consideration that $4 N Q O$ requires metabolic activation to be converted to the ultimate carcinogen, 4-acetoxyaminoquinoline 1-oxide, which reacts with DNA and causes genetic damage through formation of alkali labile sites and single strand breaks, absorption of this compound and subsequent conversion by cytochrome P450 proteins to xenobiotics metabolites can occur in gingival epithelia. ${ }^{23}$ To the best of our knowledge, there are no studies that investigated

Table 2. Incidence of histopathological lesions in tongue of rats in the 4-nitroquinoline 1-oxide (4NQO) model for oral carcinogenesis.

\begin{tabular}{|c|c|c|c|c|c|}
\hline \multirow{2}{*}{$\begin{array}{l}\text { Groups } \\
\text { (week) }\end{array}$} & \multirow{2}{*}{$\begin{array}{c}\text { No. of } \\
\text { animals }\end{array}$} & \multicolumn{4}{|c|}{ Lesions } \\
\hline & & Normal & Hyperplasia & Dysplasia & Carcinoma \\
\hline 0 (Control) & 10 & 10 & 0 & 0 & 0 \\
\hline 4 & 10 & 10 & 0 & 0 & 0 \\
\hline 12 & 10 & 0 & 7 & 3 & 0 \\
\hline 20 & 10 & 0 & 0 & 3 & 7 \\
\hline
\end{tabular}

a 4 NQO - 50 ppm by drinking water

Table 3. Histopathological pattern of gingiva following 4-nitroquinoline 1-oxidea administration.

\begin{tabular}{lccccc}
\hline Groups & \multirow{5}{c}{$\begin{array}{c}\text { Le of } \\
\text { lweek) }\end{array}$} & \multicolumn{5}{c}{ Lesions } \\
\cline { 3 - 6 } & & Normal & Hyperplasia & Dysplasia & Carcinoma \\
\cline { 3 - 6 } 0 (Control) & 10 & 10 & 0 & 0 & 0 \\
4 & 10 & 10 & 0 & 0 & 0 \\
12 & 10 & 10 & 0 & 0 & 0 \\
20 & 10 & 3 & 5 & 2 & 0 \\
\hline
\end{tabular}

a 4 NQ - 50 ppm by drinking water 
metabolization of $4 \mathrm{NQO}$ on gingival tissue. This requires further study. Thus far, the junctional epithelium of molar-tooth, with high proliferative and metabolic activity, may change pathologically and constitute the earliest gingival change induced by $4 \mathrm{NQO}$. Our results demonstrated that $4 \mathrm{NQO}$ was able to induce gingival changes as depicted by squamous gingival hyperplasia after 20 weeks following chronicle administration. Additionally, some animals pointed out squamous epithelium displaying cellular atypia characterized by abnormally differentiated squamous layers usually accompanied by thickening of the epithelium. This lesion was categorized as epithelial dysplasia having the potential to progress to squamous cell tumours in oral cavity. ${ }^{9}$ Taken as a whole, we assumed, therefore, that $4 \mathrm{NQO}$ was able to induce rat gingival changes following tongue carcinogenesis. Nevertheless, gingival carcinomas were not found in this setting. It is important to stress that the development of tongue carcinoma following $4 \mathrm{NQO}$ intake was localized mainly to the dorsum of the tongue. When animals were consuming the carcinogen in drinking water, the $4 \mathrm{NQO}$ solution might have had intimate contact with the mucosa of the dorsum of the tongue, where it could have had a direct carcinogenic effect. This may explain the effectiveness in the tongue mucosa in this tumor producing test system. Conversely, it is conceivable that exposure to $4 \mathrm{NQO}$ for a longer period of time may have eventually induced squamous cell carcinomas. Considering the end-point adopted of this study (20 weeks), a strong decrease in weight gain was noticed in the experimental group being animals deteriorated with subsequent cachexia. In this sense, we postulated that longer experimental design is not feasible for studying rat gingival carcinomas.

\section{CONCLUSIONS}

Taken together, our results support the notion that $4 \mathrm{NQO}$ is more effective in rat tongue mucosa than gingival tissue. Probably, this discrepancy depends strongly on route of administration and the susceptibility with respect to animals species. Certainly, such data will contribute when using this experimental test-system for understanding oral cancer pathogenesis. Applicability to clinical practice for persons at high risk of oral cancer, such as in smokers or in alcoholism, as well as patients diagnosed with oral dysplasia or carcinoma, however, remains to be developed.

\section{ACKNOWLEDGMENTS}

The authors are grateful to Paulo Cesar Cardoso and Maria Luiza Falaguera Ardanaz for their technical assistance. This work was supported by FAPESP (Fundação de Amparo à Pesquisa do Estado de São Paulo).

\section{REFERENCES}

1. Magrath I, Litvak J. Cancer in developing countries: opportunity and challenge. J Natl Cancer Inst 1993;85:862-874.

2. Xi S, Grandis JR. Gene therapy for the treatment of oral squamous cell carcinoma. J Dent Res 2003;82:11-16.

3. Hahn M, Hagedorn G, Kuhlisch E, Schackert HK, Edkelt U Genetic polymorphisms of drug-metabolizing enzymes and susceptibility to oral cavity cancer. Oral Oncol 2002;38:486490.

4. Kitakawa D, Cabral LAG, Marques ME, Salvadori DM, Ribeiro DA. Medium-term tongue carcinogenesis assays: a comparative study between 4-nitroquinoline 1-oxide (4NQO) induced rat and dimethylbenzanthracene (DMBA) induced hamster carcinogenesis. J Exp Animal Sci 2006;43:219227.

5. Tanaka T, Kohno H, Sakata K, Yamada Y, Hirose Y, Sugie S, Mori H. Modifying effects of dietary capsaicin and rotenone on 4-nitroquinoline 1-oxide-induced rat tongue carcinogenesis. Carcinogenesis 2002;23:1361-1367.

6. Ohne M, Satoh T, Yamada S, Takai H. Experimental tongue carcinoma of rats induced by oral administration of 4-nitroquinoline 1-oxide (4NQO) in drinking water. Oral Surg Oral Med Oral Pathol 1985;59:600-607.

7. Nishimura A. Changes in $\mathrm{Bcl}-2$ and Bax expression in rat tongue during 4-nitroquinoline 1-oxide-induced carcinogenesis. J Dent Res 1999;78:1264-1269.

8. Niwa S, Ueno S, Shirasu R. Alteration of pRB expression in the development of rat tongue carcinoma induced by 4nitroquinoline 1-oxide. Oral Oncol 2001;37:579-585.

9. Okazaki Y, Tanaka Y, Tonogi M, Yamane G. Investigation of environmental factors for diagnosing malignant potential in oral epithelial dysplasia. Oral Oncol 2002;38:562-573.

10. Vered M, Daniel N, Hirshberg A, Dayan D. Histomorphologic and morphometric changes in minor salivary glands of the rat tongue during 4-nitroquinoline 1-oxide-induced carcinogenesis. Oral Oncol 2003;39:491-496.

11. Tang XH, Knudsen B, Benis D, Tickoo S, Gudas LJ. Oral cavity and esophageal carcinogenesis modeled in carcinogentreated mice. Clin Cancer Res 2004;10:301-303. 
12. Kramer IR, Lucas RB, Pindbord JJ. Definition of leukoplakia and related lesions: an aid to studies on oral precancer. Oral Surg Oral Med Oral Pathol 1978;46:518-539.

13. Nauta JM, Roodenberg JLN, NIkkels PGJ, Witjes MJH, Vermey A. Comparison of epithelial dysplasia - the $4 \mathrm{NQO}$ rat palate model and human oral mucosa. Int J Oral Maxillofac Surg 1995;24:53-58.

14. Ribeiro DA, Silva RN, Darros BR, Salvadori DM, Marques ME. Genomic instability in non-neoplastic oral mucosa cells can predict risk during 4-nitroquinoline 1-oxide induced rat tongue carcinogenesis. Oral Oncol 2004;40:910915.

15. Ribeiro DA, Salvadori DM, Marques ME. Abnormal expression of bcl-2 and bax during the development of squamous cell carcinoma induced by 4-nitroquinoline 1-oxide. Int $J$ Exp Pathol 2005;86:375-381.

16. Neville, Brad W.; Damm, Douglas D. Patologia Oral \& Maxilofacial, Rio de Janeiro: Guanabara Koogan 2 Ed. 2004 [in Portuguese].

17. Brunet L, Miranda J, Farre M, Berini L, Mendieta C. Gingival enlargement induced by drugs. Drug Saf 1996;15:219231.

18. Haschek WM, Rousseaux CG. Oral mucosa. In: Fundamentals of Toxicology Pathology. Academic Press, San Diego, 1998.

19. Ten Cate AR. Oral Histology: development, structure and function, $5^{\text {th }}$ ed. Mosby-Year book Inc, St Louis, USA, 1998.

20. Shojaei $A H$. Buccal mucosa as a route for systemic drug delivery: a review. J Pharm Pharmaceut Sci 1998;1:15-30.

21. Shimono M, Ishikawa T, Enokiya YM, Muramatsu T, Matsuzaka K, Inoue T, Abiko Y, Yamaza T, Kido MA, Tanaka T, Hashimoto S. Biological characteristics of the junction epithelium. J Electron Microsc 2003;52:627-639.

22. Watanabe K, Petro BJ, Sevandal M, Anshuman S, Jovanovic A, Tyner AL. Histochemical examination of periodontal junctional epithelium in p21/p27 double knockout mice. Eur J Oral Sci 2004;112:253-258.

23. Vondracek M, Xi Z, Larsson $P$, Baker V, Mace K, Pfeifer A, Tjalve H, Donato MJ, Gomez-Lechon MJ, Grafstrom RC. Cytochrome $\mathrm{P} 450$ expression and related metabolism in human buccal mucosa. Carcinogenesis 2001;22:481-488. 